\title{
Lifestyle and Clinical Risk Factors for Incident Rheumatoid Arthritis-associated Interstitial Lung Disease
}

\author{
Vanessa L. Kronzer ${ }^{1}$ iD, Weixing Huang ${ }^{2}$, Paul F. Dellaripa ${ }^{2}$, Sicong Huang ${ }^{\text {(D) }, ~ V i v i ~ F e a t h e r s ~}{ }^{2}$, Bing Lu², \\ Christine K. Iannaccone ${ }^{3}$, Ritu R. Gill ${ }^{4}$, Hiroto Hatabu ${ }^{5}$, Mizuki Nishino ${ }^{6}$, Cynthia S. Crowson ${ }^{7}$ (D), \\ John M. Davis III ${ }^{1}$, Michael E. Weinblatt ${ }^{2}$, Nancy A. Shadick ${ }^{2}$ ID, Tracy J. Doyle ${ }^{8}$, and Jeffrey A. Sparks ${ }^{2}$ iD
}

\begin{abstract}
Objective. To determine the association between novel lifestyle factors on risk of rheumatoid arthritis (RA)-associated interstitial lung disease (ILD), define the threshold at which smoking increases RA-ILD risk, and calculate the degree to which known lifestyle and clinical factors predict RA-ILD.

Methods. This nested case-control study matched incident RA-ILD cases to RA non-ILD controls on age, sex, RA duration, rheumatoid factor, and time from exposure assessment to RA-ILD. Exposures included education, BMI, smoking, anticyclic citrullinated peptide antibodies, race, joint erosions, rheumatoid nodules, C-reactive protein (CRP), disease activity score, functional status, disease-modifying antirheumatic drug use, and glucocorticoid use. OR for each exposure on risk of RA-ILD were obtained from logistic regression models. Area under the curve (AUC) was calculated based on all lifestyle and clinical exposures.

Results. We identified 84 incident RA-ILD cases and 233 matched controls. After adjustment, obesity, high-positive CRP ( $\geq 10 \mathrm{mg} / \mathrm{L}$ ), and poor functional status (multidimensional Health Assessment Questionnaire $[\mathrm{MDHAQ}] \geq 1$ ) were associated with increased risk of RA-ILD (OR 2.42, 95\% CI 1.11-5.24 vs normal BMI; OR 2.61, 95\% CI 1.21-5.64 vs CRP < 3 mg/L; OR 3.10, 95\% CI 1.32-7.26 vs MDHAQ < 0.2). Smoking 30 pack-years or more was strongly associated with risk of RA-ILD compared to never smokers (OR 6.06, 95\% CI 2.72-13.5). Together, lifestyle and clinical risk factors for RA-ILD had an AUC of 0.79 (95\% CI 0.73-0.85).

Conclusion. Obesity, CRP, functional status, and extensive smoking may be novel risk factors for RA-ILD that may be useful for RA-ILD risk assessment and prevention. The overall ability to predict RA-ILD remains modest.
\end{abstract}

Key Indexing Terms: interstitial lung disease, obesity, respiratory diseases, rheumatoid arthritis

Approximately 5-10\% of patients with rheumatoid arthritis (RA) have clinically significant RA-associated interstitial lung disease (ILD), and an additional 20-30\% may have subclinical RA-ILD ${ }^{1,2,3}$. Further, RA-ILD is associated with a median survival of fewer than 3 years after diagnosis ${ }^{2}$, with $2-10$ times the odds of mortality compared to RA patients without ILD ${ }^{4,5}$. Identifying predictors of
RA-ILD is crucial to better understand the underlying pathogenesis and to allow for earlier diagnosis and treatment, with the goal of preventing irreversible lung damage. In the future, ascertaining predictors of RA-ILD may even build the foundation for interventions that can improve its poor survival by early detection or altering the natural history prior to clinically severe RA-ILD.
This work was supported by the Rheumatology Research Foundation $K$ Supplement Award; National Institutes of Health (NIH; grant numbers K23 AR069688, K23 HL119558, R03 AR075886, R03 HL148484, L30 AR066953, P30 AR070253, and P30 AR072577); and the R. Bruce and Joan M. Mickey Research Scholar Fund. The Brigham Rheumatoid Arthritis Sequential Study is funded by grants from Bristol-Myers Squibb, Crescendo Bioscience, and Sanofi. The funders had no role in study design, data collection, analysis, decision to publish, or preparation of the manuscript. The content is solely the responsibility of the authors and does not necessarily represent the official views of Harvard University, its affiliated academic bealth care centers, or the NIH.

${ }^{1}$ V.L. Kronzer, MD, MSCI, J.M. Davis III, MD, MS, Division of Rheumatology, Mayo Clinic, Rochester, Minnesota ${ }^{2}$ W. Huang, MSPH, P.F. Dellaripa, $M D$, S. Huang, MD, MS, V. Feathers, MS, B. Lu, MD, DrPH, M.E. Weinblatt, MD, N.A. Shadick, MD, MPH, J.A. Sparks, MD, $M M S c$, Division of Rheumatology, Inflammation, and Immunity, Brigham and Women's Hospital and Harvard Medical School, Boston;

${ }^{3}$ C.K. Iannaccone, MPH, Division of General Internal Medicine, Brigham and Women's Hospital, Boston; ${ }^{4}$ R.R. Gill, MD, MPH, Department of Radiology, Harvard Medical School, Beth Israel Deaconess Medical Center, Boston; ${ }^{5}$ H. Hatabu, MD, PhD, Department of Radiology, Brigham and Women's Hospital, Boston; ${ }^{6} M$. Nishino, MD, Department of Radiology, Brigham and Women's Hospital, and Department of Imaging, Dana-Farber Cancer Institute, Boston; ${ }^{7}$ C.S. Crowson, PhD, Division of Rheumatology, Mayo Clinic, Rochester, and Department of Health Sciences Research, Mayo Clinic, Rochester, Minnesota; ${ }^{8}$ T.J. Doyle, MD, MPH, Division of Pulmonary and Critical Care; Brigham and Women's Hospital and Harvard Medical School, Boston, Massachusetts, USA.

T.J. Doyle and J.A. Sparks contributed equally to this manuscript.

The authors declare no conflicts of interest.

Address correspondence to Dr. J.A. Sparks, 60 Fenwood Road, Boston, MA 02115,USA.Email:jsparks@bwh.harvard.edu.

Full Release Article. For details see Reprints and Permissions at jrheum.org. Accepted October 23, 2020. 
There are several gaps in the RA-ILD literature. First, no studies have investigated the association between lifestyle factors such as education or obesity on RA-ILD risk, despite evidence that they are risk factors for $\mathrm{RA}^{6,7,8,9}$. Second, while many studies have shown smoking to be a risk factor for RA-ILD ${ }^{10-16}$, a few have not found an association ${ }^{17,18,19}$, possibly due to a threshold effect for smoking on RA-ILD risk. One study showed that smoking over 25 pack-years was associated with a dramatic increase in physiologic and radiologic abnormalities suggestive of RA-ILD compared to fewer than 25 pack-years, but this study did not explore additional smoking exposure thresholds or stratification groups, and it used pulmonary function abnormalities as the outcome instead of RA-ILD ${ }^{10}$. Third, existing studies of RA-ILD risk have typically lacked comprehensive adjustment for many potential confounders ${ }^{1,5,10-29}$, resulting in discordant results for certain risk factors including male sex, RA duration, rheumatoid factor (RF) positivity, disease activity, functional status, prednisone use, and rheumatoid nodules. Further, the degree to which known lifestyle and clinical risk factors together predict risk of RA-ILD remains unknown.

To address these gaps, our objectives were 3-fold: (1) To elucidate the association of lifestyle factors such as education and obesity with subsequent risk of RA-ILD; (2) to define the pack-years threshold at which smoking increases risk of RA-ILD; and (3) to determine the degree to which a comprehensive assessment of known risk factors for RA-ILD predicts disease development. We hypothesized that lower education, obesity, and smoking over a threshold of 20 pack-years would be associated with increased risk of RA-ILD but that all risk factors together would have only a modest ability to predict RA-ILD, pointing to the likely existence of unmeasured risk factors.

\section{MATERIALS AND METHODS}

Study design and participants. This nested case-control study was performed within the Brigham Rheumatoid Arthritis Sequential Study (BRASS), an ongoing large, single-center, prospective registry of patients with RA since $2003^{30}$. With its low patient turnover, this population may have reasonable generalizability to other RA populations. For example, the mean age (57 yrs), and proportions of females ( $82 \%)$, new-onset RA (20\%), and high functional status (82\%) are nearly identical to RA patients in the Consortium of Rheumatology Researchers of North America (CORRONA) registry (59\%, $75 \%, 20 \%$, and $88 \%$, respectively) $)^{30,31}$. All RA cases met 1987 American College of Rheumatology (ACR) or 2010 ACR/European League Against Rheumatism criteria as determined by a rheumatologist ${ }^{32,33}$, and those with verified RA were eligible to participate ${ }^{30}$. Blood samples, RA characteristics, and physician/patient-reported measures were collected at enrollment and semiannually ${ }^{30}$. This BRASS substudy received approval from the Partners HealthCare Institutional Review Board (2016P000226), followed STrengthening the Reporting of OBservational studies in Epidemiology (STROBE) guidelines for observational studies, and complied with the Declaration of Helsinki.

RA-ILD cases and RA non-ILD controls. High-resolution chest computed tomography (CT) scans of BRASS participants performed for clinical care up until April 14, 2016, were reviewed by 2 attending thoracic radiologists and 1 attending pulmonologist. RA-ILD was defined as presence of interstitial lung abnormalities on the research read of chest CT, as described previously ${ }^{28}$. In a previous study examining the same RA-ILD cases used in this analysis, the clinical significance of CT-defined ILD was found to be high in several ways. First, of 78 RA-ILD cases for whom clinical radiographic reports were available, 68 (87\%) had features consistent with RA-ILD noted on the report. Second, 64 (75\%) of the 85 RA-ILD cases had additional clinical evaluation and/or follow-up for the condition. In addition, 32 (38\%) had changes to RA medications after CT scan. Finally, 32 (38\%) of the RA-ILD cases died during study follow-up compared to only $126(9 \%)$ in the previously analyzed RA population ${ }^{28}$. Controls were BRASS participants with absence of patient-reported ILD and absence of both billing codes and physician-reported history of asthma, chronic obstructive pulmonary disease, pulmonary fibrosis, bronchiectasis, bronchiolitis obliterans-organizing pneumonia, drug-induced pneumonitis, or tuberculosis. We did not require that all controls have chest CT performed, since doing so could introduce selection bias due to the clinical indication of the imaging. Each case was matched to up to 3 controls on age (within 5 yrs), sex, RA duration (within 5 yrs), RF positivity, and time from exposure assessment to index date (within 6 months). We matched by the above-known RA-ILD risk factors to improve power to investigate possible novel associations, knowing that this would slightly decrease the model's ability to predict RA-ILD. Index date was the date of RA-ILD by the initial CT chest for the case and matched date for corresponding controls.

Exposures and covariates. To ensure that assessment of exposures preceded the date of ILD, we identified the earliest study visit date containing these data occurring before the index date. Exposure and covariate variables obtained from BRASS study visits included age, sex, RA duration, race/ ethnicity (white, non-Hispanic vs other), education (lower than college degree vs higher), joint erosions (present vs absent on hand plain radiographs), rheumatoid nodules (present vs absent), BMI categories, smoking status (never, past, or current), smoking pack-years, disease activity score with 28-joint counts based on C-reactive protein (DAS28-CRP; remission $[<2.6]$, low $[2.6$ to $<3.2]$, moderate $[3.2$ to $<5.2]$, or high $[\geq 5.1])^{34}$, functional status by multidimensional Health Assessment Questionnaire (MDHAQ; 0 to $<0.2,0.2<1.0$, or $\geq 1.0)^{35}$, and biologic disease-modifying antirheumatic drug (bDMARD), methotrexate (MTX), or prednisone use (never, past, current). RF positivity, anticyclic citrullinated peptide (anti-CCP) positivity, and CRP (normal $[<3 \mathrm{mg} / \mathrm{L}$ ], low-positive [3 to $<10 \mathrm{mg} / \mathrm{L}]$, or high $[\geq 10 \mathrm{mg} / \mathrm{L}]$ ) came from study visit blood work.

Statistical analysis. We compared continuous variables using Wilcoxon rank-sum tests, and categorical variables using chi-square tests. For the association analyses, conditional logistic regression models of each exposure were fitted to obtain OR with $95 \%$ CI for RA-ILD case status, adjusting for anti-CCP, race/ethnicity, education, BMI (continuous), and smoking pack-years (continuous). Since disease activity and treatment closely interact, the main models of these factors did not adjust for each other. Exploratory sensitivity analyses added prednisone and MTX use to the disease activity model, and disease activity to each drug model, in order to investigate possible independent associations. For ordinal variables, we calculated $P$ for trend by first calculating the median value within each category and then using those values as a continuous variable in a separate model. To determine the degree to which all risk factors predicted RA-ILD risk, we fit receiver-operating characteristic (ROC) curves and calculated the area under the curve (AUC). The threshold for statistical significance was a 2 -sided $P<0.05$. No participants were missing any data. Analyses were prespecified in a protocol and performed using SAS version 9.4 (SAS Institute Inc.).

\section{RESULTS}

Characteristics of RA-ILD cases and controls. Of the $1100 \mathrm{RA}$ cases included in BRASS, we identified 84 cases of confirmed RA-ILD and 233 matched RA non-ILD controls. Among controls, $60(26 \%)$ had at least 1 chest CT performed, and an additional $76(33 \%)$ had at least 1 chest radiograph. Therefore, the majority (58\%) of controls had chest imaging performed 
that did not clinically reveal RA-ILD. Characteristics of controls with CT compared to without CT were similar, with none of the factors associated with RA-ILD being associated with receipt of chest $\mathrm{CT}$ in controls, including education (Supplementary Table 1 , available with the online version of this article). The median time between BRASS study visit where exposures/covariates were assessed and the index date of RA-ILD (or matched date) was 1.5 (IQR 0.6-2.5) years for RA-ILD cases and 1.9 (IQR 1.6-5.6) years for controls. At the time of exposure assessment, RA-ILD cases had more rheumatoid nodules, greater history of smoking, higher CRP, higher disease activity (DAS28-CRP), worse functional status (MDHAQ), less bDMARD and MTX use, and more prednisone use when compared to controls based on univariate analyses (Table 1).

Education and obesity. After further adjusting for anti-CCP, race, and smoking, we found that participants with lower education had a decreased risk of RA-ILD compared to participants with a college education or higher (Table 2). Obesity (BMI $\geq 30 \mathrm{~kg} / \mathrm{m}^{2}$ ) was associated with a more than 2 -fold increased risk of RA-ILD, with evidence of a dose-response relationship $(P$ for trend 0.02; Table 2).

Smoking threshold. After adjustment, cumulative past smoking was not associated with RA-ILD (Table 3). However, current smoking was associated with a more than 3 -fold increase in odds of RA-ILD compared to never smoking (Table 3). Further, smoking 30 pack-years or more was associated with a 6-fold increased risk compared to nonsmokers $(P$ for trend $<0.001$; Table 3). In contrast, smoking levels $<30$ pack-years were not associated with increased risk of RA-ILD (Table 3).

Other risk factors for RA-ILD. Other factors that remained statistically significant predictors of RA-ILD after adjustment included high-positive CRP, moderate or high disease activity (DAS28-CRP 3.2 or higher), poor functional status (MDHAQ $\geq 1.0$ ), and current prednisone use, while past or current MTX use was associated with decreased risk of RA-ILD (Table 4). A sensitivity analysis adjusting disease activity for prednisone and MTX use showed that moderate and high disease activity remained statistically significant predictors of RA-ILD (OR 2.22, 95\% CI 1.04-4.72 and OR 5.09, 95\% CI $1.58-16.4)$, respectively, compared to remission $(P$ for trend $0.003)$. Similarly, the association between prednisone and RA-ILD remained significant even after additionally adjusting for disease activity (OR 4.08, 95\% CI 1.23-13.6).

Ability to predict RA-ILD. Together, these known demographics (age, race, education), lifestyle (BMI, smoking pack-yrs), and RA-related (RA duration, RF positivity, anti-CCP positivity, joint erosions, rheumatoid nodules, bDMARD use, MTX use, prednisone use, disease activity, functional status) risk factors explain a modest amount of an individual's risk for RA-ILD (Figure 1). The AUC for all factors combined was 0.79 (95\% CI 0.73-0.85).

\section{DISCUSSION}

This case-control study discovered several novel predictors for incident RA-ILD, including obesity, higher education level,
Table 1. Characteristics of the 84 incident RA-ILD cases and their 233 RA non-ILD controls. These characteristics were collected at the time of the earliest BRASS study visit.

\begin{tabular}{|c|c|c|c|}
\hline & $\begin{array}{l}\text { RA-ILD cases, } \\
\quad \mathrm{n}=84\end{array}$ & $\begin{array}{l}\text { RA controls, } \\
\mathrm{n}=233\end{array}$ & $P$ \\
\hline $\begin{array}{l}\text { Years to index/matched date, } \\
\text { median (IQR) }\end{array}$ & $1.5(0.6-2.5)$ & $1.9(1.6-5.6)$ & $0.84^{\mathrm{a}}$ \\
\hline Age, yrs, mean (SD) & $67(10)$ & $66(11)$ & $0.41^{a}$ \\
\hline Female sex & $65(77)$ & $186(80)$ & $0.64^{\mathrm{a}}$ \\
\hline RA duration, yrs, mean (SD) ${ }^{b}$ & $20(12)$ & $20(11)$ & $0.99^{a}$ \\
\hline RF-positive & $73(87)$ & $195(84)$ & $0.48^{\mathrm{a}}$ \\
\hline CCP-positive & $70(83)$ & $190(82)$ & 0.71 \\
\hline White, non-Hispanic & $76(90)$ & $219(94)$ & 0.28 \\
\hline Education, below college & $30(36)$ & $98(42)$ & 0.31 \\
\hline Joint erosions & $49(58)$ & $144(62)$ & 0.58 \\
\hline Rheumatoid nodules & $42(50)$ & $80(34)$ & 0.01 \\
\hline $\mathrm{BMI}, \mathrm{kg} / \mathrm{m}^{2}$ & & & 0.18 \\
\hline$<20$ & $4(5)$ & $16(7)$ & \\
\hline 20 to $<25$ & $20(23)$ & $77(33)$ & \\
\hline 25 to $<30$ & $32(38)$ & $87(37)$ & \\
\hline$\geq 30$ & $28(33)$ & $53(23)$ & \\
\hline Smoking status & & & 0.03 \\
\hline Never & $33(39)$ & $124(53)$ & \\
\hline Past & $43(51)$ & $100(43)$ & \\
\hline Current & $8(10)$ & $9(4)$ & \\
\hline Smoking, pack-yrs & & & $<0.001$ \\
\hline Never smoker & $36(43)$ & $126(54)$ & \\
\hline $1-9$ & $7(8)$ & $45(19)$ & \\
\hline $10-19$ & $11(13)$ & $28(12)$ & \\
\hline $20-29$ & $7(8)$ & $21(9)$ & \\
\hline$\geq 30$ & $23(27)$ & $13(6)$ & \\
\hline CRP, mg/L & & & 0.006 \\
\hline $\operatorname{Normal}(<3)$ & $33(39)$ & $136(58)$ & \\
\hline Low-positive $(3$ to $<10)$ & $31(37)$ & $67(29)$ & \\
\hline High-positive $(\geq 10)$ & $20(24)$ & $30(13)$ & \\
\hline DAS28-CRP & & & $<0.001$ \\
\hline Remission $(<2.6)$ & $27(32)$ & $104(45)$ & \\
\hline Low $(2.6$ to $<3.2)$ & $4(5)$ & $39(17)$ & \\
\hline Moderate $(3.2$ to $<5.1)$ & $37(44)$ & $70(30)$ & \\
\hline $\operatorname{High}(\geq 5.1)$ & $16(19)$ & $20(9)$ & \\
\hline MDHAQ Score & & & $<0.001$ \\
\hline 0 to $<0.2$ & $15(18)$ & $64(27)$ & \\
\hline $0.2<1.0$ & $37(44)$ & $127(55)$ & \\
\hline$\geq 1.0$ & $32(38)$ & $42(18)$ & \\
\hline bDMARD use & & & 0.03 \\
\hline Never & $29(35)$ & $76(33)$ & \\
\hline Past & $19(23)$ & $27(12)$ & \\
\hline Current & $36(43)$ & $130(56)$ & \\
\hline Methotrexate use & & & 0.03 \\
\hline Never & $18(21)$ & $24(10)$ & \\
\hline Past & $32(38)$ & $93(40)$ & \\
\hline Current & $34(41)$ & $116(50)$ & \\
\hline Prednisone use & & & 0.007 \\
\hline Never & $5(6)$ & $31(13)$ & \\
\hline Past & $42(50)$ & $140(60)$ & \\
\hline Current & $37(44)$ & $62(27)$ & \\
\hline
\end{tabular}

Values are $\mathrm{n}(\%)$ unless otherwise indicated. ${ }^{\mathrm{a}}$ Matched factor. ${ }^{\mathrm{b}}$ Range was $1-46$ years for cases and 2-47 years for controls. Median (IQR) was $20(10-31)$ for cases and 18 (11-29) for controls. bDMARD: biological disease-modifying antirheumatic drug; BRASS: Brigham Rheumatoid Arthritis Sequential Study; CCP: citrullinated peptide antibody; CRP: C-reactive protein; DAS28: Disease Activity Score in 28 joints; ILD: interstitial lung disease; MDHAQ: multidimensional Health Assessment Questionnaire; RA: rheumatoid arthritis; RF: rheumatoid factor. 
Table 2. Association between education, obesity, and incident RA-ILD. These data came from the 84 BRASS RA-ILD cases and 233 RA non-ILD controls.

\section{OR Conditioned on Multivariable ${ }^{\mathrm{b}}$ OR Matching Factors ${ }^{\mathrm{a}} \quad(95 \% \mathrm{CI})$ \\ $(95 \% \mathrm{CI})$}

\begin{tabular}{|c|c|c|}
\hline Education lower than college & $0.76(0.46-1.26)$ & $0.53(0.30-0.95)$ \\
\hline \multicolumn{3}{|l|}{$\mathrm{BMI}, \mathrm{kg} / \mathrm{m}^{2}$} \\
\hline$<20$ & $0.98(0.30-3.21)$ & $1.00(0.29-3.43)$ \\
\hline 20 to $<25$ & (ref) & (ref) \\
\hline 25 to $<30$ & $1.35(0.72-2.52)$ & $1.56(0.79-3.06)$ \\
\hline$\geq 30$ & $2.05(1.03-4.07)$ & $2.42(1.11-5.24)$ \\
\hline
\end{tabular}

Bold values indicate statistical significance to $P<0.05$. ${ }^{\text {a }}$ All models were conditioned on matching factors (age, sex, RA duration, RF status, and time from study visit to index date). ${ }^{\text {b }}$ Also adjusting for anti-CCP, race/ ethnicity, education (in BMI model), BMI (in education model), and smoking pack-years. Anti-CCP: anticyclic citrullinated peptide antibody; BRASS: Brigham Rheumatoid Arthritis Sequential Study; ILD: interstitial lung disease; RA: rheumatoid arthritis.

Table 3. Association between smoking and incident RA-ILD. These data came from the 84 BRASS RA-ILD cases and 233 RA non-ILD controls.

$\begin{array}{cc}\text { OR Conditioned on } & \text { Multivariable } \mathrm{e}^{\mathrm{b}} \text { OR } \\ \text { Matching Factors } & (95 \% \mathrm{CI}) \\ (95 \% \mathrm{CI}) & \end{array}$

\begin{tabular}{lcc}
\hline $\begin{array}{l}\text { Smoking status } \\
\text { Never }\end{array}$ & $(\mathrm{ref})$ & $(\mathrm{ref})$ \\
Past & $1.56(0.92-2.63)$ & $1.68(0.97-2.92)$ \\
$\quad$ Current & $\mathbf{2 . 9 1 ( 1 . 0 8 - 7 . 8 5 )}$ & $\mathbf{3 . 2 7}(\mathbf{1 . 1 5}-\mathbf{9 . 2 9})$ \\
Smoking, pack-yrs & & \\
$\quad$ Never smoker & $(\mathrm{ref})$ & $(\mathrm{ref})$ \\
$1-9$ & $0.53(0.22-1.26)$ & $0.54(0.22-1.31)$ \\
$10-19$ & $1.30(0.59-2.89)$ & $1.42(0.62-3.24)$ \\
$20-29$ & $1.16(0.42-3.19)$ & $1.44(0.51-4.06)$ \\
$\geq 30$ & $\mathbf{5 . 3 4}(\mathbf{2 . 5 0}-\mathbf{1 1 . 5})$ & $\mathbf{6 . 0 6}(\mathbf{2 . 7 2 - 1 3 . 5})$ \\
\end{tabular}

Bold values indicate statistical significance to $P<0.05$. ${ }^{\text {a All models were }}$ conditioned on matching factors (age, sex, RA duration, RF status, and time from study visit to index date). ${ }^{\mathrm{b}}$ Also adjusting for anti-CCP, race/ ethnicity, education, and BMI. Anti-CCP: anticyclic citrullinated peptide antibody; BRASS: Brigham Rheumatoid Arthritis Sequential Study; ILD: interstitial lung disease; RA: rheumatoid arthritis; RF: rheumatoid factor.

high-positive CRP, and poor functional status. We also made the discovery that smoking beyond a threshold of 30 pack-years had a strong association with RA-ILD, while there was no evidence of an association for lower levels of smoking. Third, we confirmed existing RA-ILD risk factors such as disease activity, suggesting disease control could reduce RA-ILD risk, and prednisone, which should be used with caution in patients with RA who are at risk for RA-ILD. Finally, we demonstrated that currently known clinical predictors of RA-ILD have only a modest ability to predict RA-ILD. These findings may shed light on the potential pathogenesis of RA-ILD, could improve patient-specific risk assessment and prevention efforts, and highlight the need for further studies on risk factors for RA-ILD.
Table 4. Association between previously studied risk factors and incident RA-ILD. These data came from the 84 BRASS RA-ILD cases and 233 RA non-ILD controls.

$\begin{array}{cc}\text { OR Conditioned on } & \text { Multivariable } \mathrm{e}^{\mathrm{b}} \text { OR } \\ \text { Matching Factors }^{\mathrm{a}} & (95 \% \mathrm{CI})\end{array}$

$(95 \% \mathrm{CI})$

\begin{tabular}{|c|c|c|}
\hline White, non-Hispanic & $0.61(0.24-1.54)$ & $0.57(0.20-1.60)$ \\
\hline Joint erosions & $0.79(0.45-1.40)$ & $0.74(0.41-1.34)$ \\
\hline Rheumatoid nodules & $2.02(1.15-3.55)$ & $1.66(0.89-3.07)$ \\
\hline \multicolumn{3}{|l|}{$\mathrm{C}$-reactive protein, $\mathrm{mg} / \mathrm{L}$} \\
\hline $\operatorname{Normal}(<3)$ & $($ ref $)$ & (ref) \\
\hline Low-positive $(3$ to $<10)$ & $1.86(1.04-3.34)$ & $1.67(0.87-3.20)$ \\
\hline High-positive $(\geq 10)$ & $2.79(1.36-5.69)$ & $2.61(1.21-5.64)$ \\
\hline$P$ for trend & 0.008 & 0.021 \\
\hline \multicolumn{3}{|l|}{ DAS28-CRP } \\
\hline Remission $(<2.6)$ & (ref) & (ref) \\
\hline Low $(2.6$ to $<3.2)$ & $0.46(0.15-1.37)$ & $0.47(0.15-1.45)$ \\
\hline Moderate $(3.2$ to $<5.1)$ & $2.28(1.21-4.30)$ & $2.13(1.09-4.19)$ \\
\hline $\operatorname{High}(\geq 5.1)$ & $4.16(1.65-10.5)$ & $4.28(1.50-12.2)$ \\
\hline$P$ for trend & $<0.001$ & 0.002 \\
\hline \multicolumn{3}{|l|}{ MDHAQ score } \\
\hline 0 to $<0.2$ & (ref) & (ref) \\
\hline $0.2<1.0$ & $1.16(0.58-2.34)$ & $1.07(0.50-2.30)$ \\
\hline$\geq 1.0$ & $3.11(1.48-6.54)$ & $3.10(1.32-7.26)$ \\
\hline$P$ for trend & $<0.001$ & 0.004 \\
\hline \multicolumn{3}{|l|}{ bDMARD use } \\
\hline Never & (ref) & $($ ref $)$ \\
\hline Past & $1.71(0.82-3.55)$ & $1.47(0.67-3.24)$ \\
\hline Current & $0.69(0.38-1.26)$ & $0.52(0.27-1.02)$ \\
\hline \multicolumn{3}{|l|}{ Methotrexate use } \\
\hline Never & (ref) & $($ ref $)$ \\
\hline Past & $0.51(0.24-1.06)$ & $0.38(0.16-0.86)$ \\
\hline Current & $0.45(0.22-0.91)$ & $0.36(0.17-0.77)$ \\
\hline \multicolumn{3}{|l|}{ Prednisone use } \\
\hline Never & (ref) & (ref) \\
\hline Past & $2.03(0.74-5.59)$ & $1.44(0.48-4.34)$ \\
\hline Current & $3.94(1.36-11.4)$ & $3.58(1.13-11.3)$ \\
\hline
\end{tabular}

Bold values indicate statistical significance to $P<0.05$. ${ }^{\text {a }}$ All models were conditioned on matching factors (age, sex, RA duration, RF status, and time from study visit to index date). ${ }^{\mathrm{b}}$ Also adjusting for anti-CCP, race/ ethnicity, education, BMI, and smoking pack-years. Anti-CCP: anticyclic citrullinated peptide antibody; bDMARD: biologic disease-modifying antirheumatic drug; BRASS: Brigham Rheumatoid Arthritis Sequential Study; CRP: C-reactive protein; DAS28: Disease Activity Score in 28 joints; ILD: interstitial lung disease; MDHAQ: multidimensional Health Assessment Questionnaire; RA: rheumatoid arthritis; RF: rheumatoid factor.

We identified obesity and higher education as possible novel lifestyle risk factors for RA-ILD. Only 1 prior study reported data on the association between BMI and RA-ILD risk and found no association ${ }^{29}$, but it had only 29 RA-ILD cases. Thus, the association between obesity and RA-ILD is a novel finding to this study. Obesity has a modest but positive association with risk of RA, supporting the biological plausibility of such an association with RA-ILD ${ }^{8,9}$. It is possible that the atelectasis or poorer-quality CT scans that occur in obese persons could result in misclassification of RA-ILD in either direction. Nevertheless, this discovery has important clinical implications, as obesity is now a second potentially modifiable 


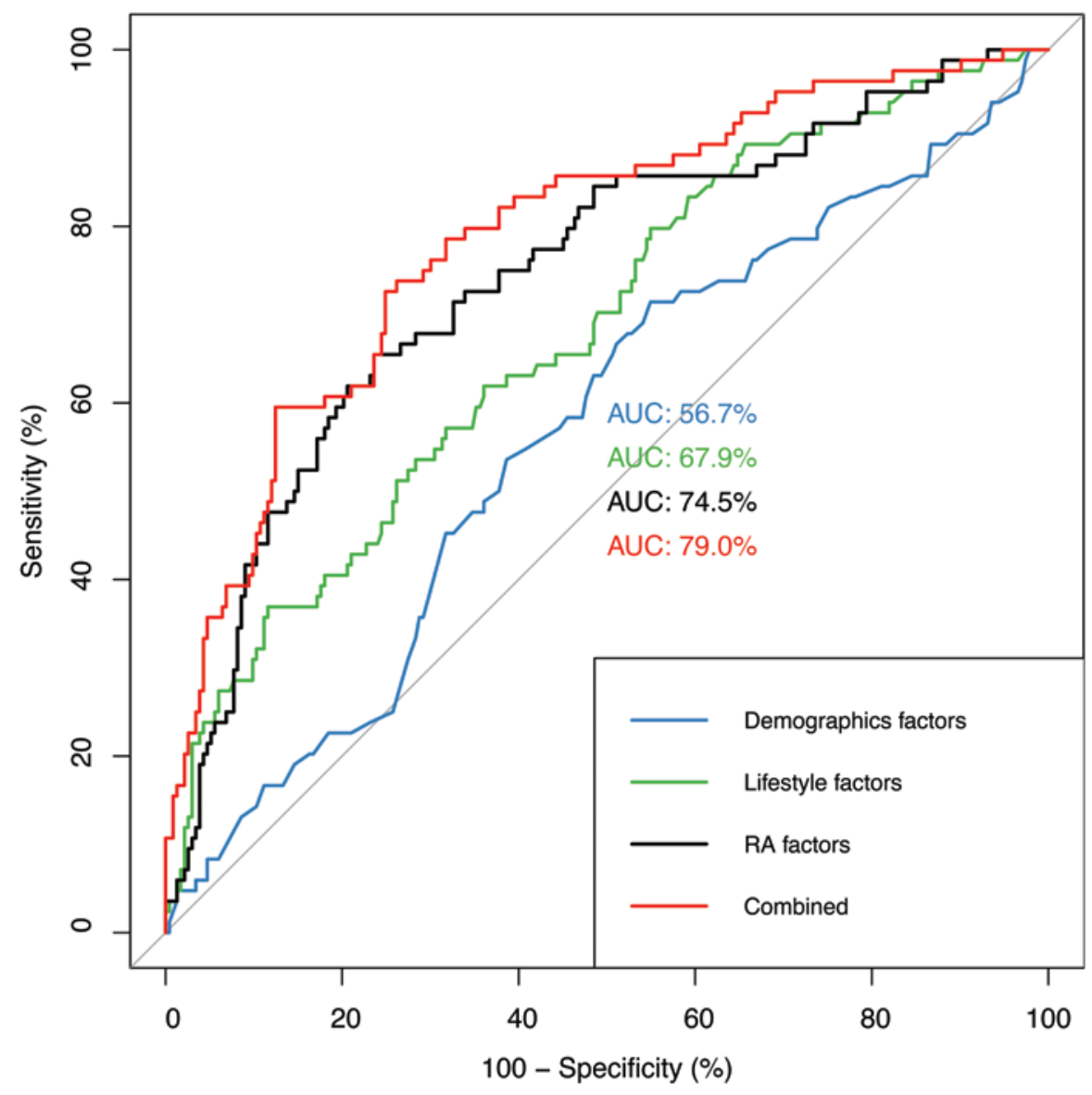

Figure 1. Receiver-operating characteristic curve for incident RA-ILD risk. These curves were fit using the 84 RA-ILD cases and 233 RA non-ILD controls in BRASS using known demographic, lifestyle, and RA clinical risk factors. AUC: area under the curve; BRASS: Brigham Rheumatoid Arthritis Sequential Study; ILD: interstitial lung disease; RA: rheumatoid arthritis.

risk factor for RA-ILD on which clinicians can counsel their patients.

The association between higher education and RA-ILD is also novel to this study. The 1 prior study of RA-ILD that reported data on education showed no association, though it was limited by small sample size ${ }^{17}$. This association between higher education and RA-ILD was surprising since higher socioeconomic status has been shown to have no association with $\operatorname{ILD}^{36}$ or $\mathrm{RA}^{37,38}$. In fact, higher education has even been associated with a lower risk of $\mathrm{RA}^{6,7}$ and other autoimmune diseases like systemic lupus erythematosus ${ }^{39}$. Thus, high medical literacy, including willingness to undergo CT imaging, may explain this association rather than a true biologic effect. Future studies should confirm the association between education and RA-ILD and investigate the mechanism for it; this may provide clues to the pathogenesis of RA-ILD. Conversely, this association may be explained by health literacy as a construct of social determinants of health.

We also identified high-positive CRP and poor functional status as novel clinical risk factors for RA-ILD. Two prior studies showed no association between CRP and RA-ILD in multivariable models, but only included CRP as a continuous variable instead of separating out high CRP from those with low or normal CRP ${ }^{18,19}$. The validity of this association is supported by the association with ESR with RA-ILD ${ }^{10,14,16,17}$. Thus, high-positive CRP may be a useful biomarker for predicting increased risk of developing RA-ILD. Similarly, we found reduced functional status (in particular, MDHAQ $\geq 1$ ) was associated with increased risk of RA-ILD. This is consistent with a prior study that showed a univariate association between poor functional status and RA-ILD ${ }^{10}$. Although 2 studies have shown no association between functional status and RA-ILD after adjusting for confounders, they were smaller than the current study $^{11,17}$. Therefore, this study showed high-positive CRP $(\geq 10$ $\mathrm{mg} / \mathrm{L}$ ) and poor functional status (MDHAQ $\geq 1$ ) to be risk factors for RA-ILD that can aid clinicians in prognosticating which patients are at increased risk for RA-ILD.

Another key finding from this study is that smoking may exhibit a threshold effect in its relationship with RA-ILD. We found that smoking 30 pack-years or more was associated with a 6-fold increase in RA-ILD risk, whereas smoking 
under this threshold was not associated with increased risk. Although most studies of RA-ILD risk show an association with smoking ${ }^{10,11,12,13,15,16}$, some others do not ${ }^{17,18,19}$. Based on our findings, a reason for this discrepancy could be that the smokers in the negative studies did not have sufficient (> 30 pack-yrs) smoking history. Alternatively, the lack of association could have resulted from lack of certain HLA-DR alleles, termed the "shared epitope," as smoking may only increase RA-ILD risk in the presence of the shared epitope ${ }^{14}$. Interestingly, this 30 pack-year threshold for RA-ILD differs from RA, where the relationship between smoking and RA risk increases at a threshold of 5-10 pack-years and plateaus at $20^{40}$. Such dissimilarity suggests that the mechanism for the association of smoking with RA differs from its association with RA-ILD. Additionally, current smokers had increased risk for RA-ILD, confirming the findings from 2 previous small cross-sectional studies ${ }^{11,29}$. Together, these results underscore the importance of smoking cessation to prevent RA-ILD, especially in patients who have not yet reached the critical 30 pack-year threshold. Future studies of RA-ILD risk should consider stratifying by pack-years rather than ever/ never smoking and investigate the pathophysiologic reasons for a threshold effect.

With its multivariable models, this study also clarified the association between several possible risk factors and RA-ILD. For example, some studies have shown a positive association for rheumatoid nodules ${ }^{10,14,16}$ and disease activity ${ }^{11,14,28}$, while other studies have shown no association between rheumatoid nodules ${ }^{11,17,28}$ or disease activity ${ }^{16,17,18}$ and risk of RA-ILD. We found that moderate to high disease activity was associated with increased risk of RA-ILD, suggesting that controlling disease activity can reduce risk. We did not find an association with rheumatoid nodules, though matching on RF may have reduced the association. Similarly, some studies have shown an association between prednisone use and RA-ILD ${ }^{14,19,21,28}$, whereas others have not ${ }^{11,13,18}$. In this study, even after accounting for disease activity, current prednisone use was associated with a 4-fold increased risk for RA-ILD. Thus, providers should use caution in prescribing prednisone in RA or even RA-ILD patients.

The lack of association with MTX use, while initially surprising given the reported association between MTX and lung disease $\mathrm{e}^{41}$, has actually been shown repeatedly in prior studies of RA-ILD risk ${ }^{11,13,14,18,19,21,28}$. In fact, 3 prior studies have even shown MTX may have a protective effect on RA-ILD risk, similar to our present study ${ }^{10,16,20}$. While it is possible that MTX may protect against RA-ILD given its therapeutic effects in RA, this decreased association might also simply represent channeling bias away from MTX use in patients with lung disease. Even so, given the lack of treatments for RA-ILD, further exploration of these 2 possibilities may be warranted.

Finally, despite discovery of these additional risk factors for RA-ILD, we found that these RA-ILD risk factors had only a modest AUC of 0.79. This finding is consistent with prior studies, whose AUC ranged from 0.60 to $0.85^{13,14,29}$, and suggests a need to identify additional risk factors for RA-ILD. Fortunately, recent studies have shown promise for biomarkers like fine-specificity anticitrullinated peptide antibodies ${ }^{11,42,43,44,45}$, matrix metalloproteinase $7^{13}$, surfactant protein $\mathrm{D}^{13,46}$, and pulmonary and activation-regulated chemokine ${ }^{13}$, as well as genetic markers such as the HLA shared epitope ${ }^{14}$ or MUC5B promoter variant ${ }^{47}$. However, these biomarkers are not usually available in routine clinical care. Further studies incorporating these biologic and genetic markers are needed.

Strengths of this study include its inclusion of numerous previously studied risk factors for RA-ILD and their prospective collection. Nevertheless, there are several important limitations. First, ILD can begin insidiously before CT is obtained, so it is possible that the predictors may have resulted from RA-ILD. Even if this were true, data were collected a median of 1.5 years before clinical detection, so these factors could still identify patients in an earlier phase in the course of RA-ILD. Second, selection bias is possible since not all BRASS participants had chest $\mathrm{CT}$ scans available for review, as these were performed for clinical purposes. For example, some controls might have had unrecognized, subclinical ILD since chest CT were performed on only $25 \%$ of controls, which would be expected to bias results toward the null. We mitigated this possibility by excluding controls with comorbidities that could be suggestive of ILD. Third, RA-ILD patients may be prone to differential follow-up, compared to patients with RA and no ILD, due to the serious nature of this condition. To mitigate any potential selection bias related to right/left censoring, our case-control study matched on time from exposure/covariate assessment to index/matched date. Fourth, matching controls on RA duration and RF positivity, while improving comparability of the groups in this study and power to detect novel associations, likely eliminated the association with anti-CCP and slightly lowered the calculated AUC since these factors were balanced between cases and controls by design. Finally, unmeasured factors exist and may be important for RA-ILD risk, such as biomarkers and genetic factors like the shared epitope and MUC5B variant.

In conclusion, we identified obesity, higher education, high-level CRP, and poor functional status as novel risk factors for RA-ILD. In addition, smoking 30 pack-years or greater was associated with a substantially increased risk of RA-ILD, whereas lower levels of smoking did not increase risk. These findings might improve our understanding of RA-ILD pathogenesis and prediction, and also suggest weight loss and smoking cessation might prevent RA-ILD. However, all known clinical risk factors for RA-ILD have only a modest ability to predict RA-ILD development. Given the poor prognosis and limited available treatment options for RA-ILD, this finding emphasizes the critical need for future studies of clinical, biological, and genetic risk factors for RA-ILD.

\section{ACKNOWLEDGMENT}

We thank Lily Martin for performing our technical review for this manuscript.

\section{ONLINE SUPPLEMENT}

Supplementary material accompanies the online version of this article.

\section{REFERENCES}

1. Gabbay E, Tarala R, Will R, Carroll G, Adler B, Cameron D, et al. 
Interstitial lung disease in recent onset rheumatoid arthritis. Am J Respir Crit Care Med 1997;156:528-35.

2. Bongartz T, Nannini C, Medina-Velasquez YF, Achenbach SJ, Crowson CS, Ryu JH, et al. Incidence and mortality of interstitial lung disease in rheumatoid arthritis: a population-based study. Arthritis Rheum 2010;62:1583-91.

3. Olson AL, Swigris JJ, Sprunger DB, Fischer A, Fernandez-Perez ER, Solomon J, et al. Rheumatoid arthritis-interstitial lung disease-associated mortality. Am J Respir Crit Care Med 2011;183:372-8.

4. Hyldgaard C, Hilberg O, Pedersen AB, Ulrichsen SP, Løkke A, Bendstrup E, et al. A population-based cohort study of rheumatoid arthritis-associated interstitial lung disease: comorbidity and mortality. Ann Rheum Dis 2017;76:1700-6.

5. Kim D, Cho SK, Choi CB, Choe JY, Chung WT, Hong SJ, et al. Impact of interstitial lung disease on mortality of patients with rheumatoid arthritis. Rheumatol Int 2017;37:1735-45.

6. Bengtsson C, Nordmark B, Klareskog L, Lundberg I, Alfredsson L. Socioeconomic status and the risk of developing rheumatoid arthritis: results from the Swedish EIRA study. Ann Rheum Dis 2005;64:1588-94.

7. Ghawi H, Crowson CS, Rand-Weaver J, Krusemark E, Gabriel SE, Juhn YJ. A novel measure of socioeconomic status using individual housing data to assess the association of SES with rheumatoid arthritis and its mortality: a population-based case-control study. BMJ Open 2015;5:e006469.

8. Crowson CS, Matteson EL, Davis JM 3rd, Gabriel SE. Contribution of obesity to the rise in incidence of rheumatoid arthritis. Arthritis Care Res 2013;65:71-7.

9. Qin B, Yang M, Fu H, Ma N, Wei T, Tang Q, et al. Body mass index and the risk of rheumatoid arthritis: a systematic review and dose-response meta-analysis. Arthritis Res Ther 2015;17:86.

10. Saag KG, Kolluri S, Koehnke RK, Georgou TA, Rachow JW, Hunninghake GW, et al. Rheumatoid arthritis lung disease. Determinants of radiographic and physiologic abnormalities. Arthritis Rheum 1996;39:1711-9.

11. Giles JT, Danoff SK, Sokolove J, Wagner CA, Winchester R, Pappas $\mathrm{DA}$, et al. Association of fine specificity and repertoire expansion of anticitrullinated peptide antibodies with rheumatoid arthritis associated interstitial lung disease. Ann Rheum Dis 2014; 73:1487-94.

12. Kelly CA, Saravanan V, Nisar M, Arthanari S, Woodhead FA, Price-Forbes AN, et al. Rheumatoid arthritis-related interstitial lung disease: associations, prognostic factors and physiological and radiological characteristics--a large multicentre UK study. Rheumatology 2014;53:1676-82.

13. Doyle TJ, Patel AS, Hatabu H, Nishino M, Wu G, Osorio JC, et al. Detection of rheumatoid arthritis-interstitial lung disease is enhanced by serum biomarkers. Am J Respir Crit Care Med 2015;191:1403-12.

14. Restrepo JF, del Rincón I, Battafarano DF, Haas RW, Doria M, Escalante A. Clinical and laboratory factors associated with interstitial lung disease in rheumatoid arthritis. Clin Rheumatol 2015;34:1529-36.

15. Zhang Y, Li H, Wu N, Dong X, Zheng Y. Retrospective study of the clinical characteristics and risk factors of rheumatoid arthritis-associated interstitial lung disease. Clin Rheumatol 2017;36:817-23.

16. Kiely P, Busby AD, Nikiphorou E, Sullivan K, Walsh DA, Creamer P, et al. Is incident rheumatoid arthritis interstitial lung disease associated with MTX treatment? Results from a multivariate analysis in the ERAS and ERAN inception cohorts. BMJ Open 2019;9:e028466.

17. Koduri G, Norton S, Young A, Cox N, Davies P, Devlin J, et al. Interstitial lung disease has a poor prognosis in rheumatoid arthritis:
Results from an inception cohort. Rheumatology 2010;49:1483-9.

18. Yin Y, Liang D, Zhao L, Li Y, Liu W, Ren Y, et al. Anti-cyclic citrullinated peptide antibody is associated with interstitial lung disease in patients with rheumatoid arthritis. PLoS One 2014;9:e92449.

19. Wang JX, Du CG. A retrospective study of clinical characteristics of interstitial lung disease associated with rheumatoid arthritis in Chinese patients. Med Sci Monit 2015;21:708-15.

20. Suissa S, Hudson M, Ernst P. Leflunomide use and the risk of interstitial lung disease in rheumatoid arthritis. Arthritis Rheum 2006;54:1435-9.

21. Wolfe F, Caplan L, Michaud K. Rheumatoid arthritis treatment and the risk of severe interstitial lung disease. Scand J Rheumatol 2007;36:172-8.

22. Inui N, Enomoto N, Suda T, Kageyama Y, Watanabe H, Chida $\mathrm{K}$. Anti-cyclic citrullinated peptide antibodies in lung diseases associated with rheumatoid arthritis. Clin Biochem 2008; 41:1074-7.

23. Sawada T, Inokuma S, Sato T, Otsuka T, Saeki Y, Takeuchi T, et al. Leflunomide-induced interstitial lung disease: prevalence and risk factors in Japanese patients with rheumatoid arthritis. Rheumatology (Oxford) 2009;48:1069-72.

24. Shidara K, Hoshi D, Inoue E, Yamada T, Nakajima A, Taniguchi A, et al. Incidence of and risk factors for interstitial pneumonia in patients with rheumatoid arthritis in a large Japanese observational cohort, IORRA. Mod Rheumatol 2010;20:280-6.

25. Mori S, Koga Y, Sugimoto M. Different risk factors between interstitial lung disease and airway disease in rheumatoid arthritis. Respir Med 2012;106:1591-9.

26. Rocha-Muñoz AD, Ponce-Guarneros M, Gamez-Nava JI, Olivas-Flores EM, Mejía M, Juárez-Contreras P, et al. Anti-cyclic citrullinated peptide antibodies and severity of interstitial lung disease in women with rheumatoid arthritis. J Immunol Res 2015;2015:151626.

27. Kurata I, Tsuboi H, Terasaki M, Shimizu M, Toko H, Honda F, et al. Effect of biological disease-modifying anti-rheumatic drugs on airway and interstitial lung disease in patients with rheumatoid arthritis. Intern Med 2019;58:1703-12.

28. Sparks JA, He X, Huang J, Fletcher EA, Zaccardelli A, Friedlander $\mathrm{HM}$, et al. Rheumatoid arthritis disease activity predicting incident clinically apparent rheumatoid arthritis-associated interstitial lung disease: a prospective cohort study. Arthritis Rheumatol 2019;71:1472-82.

29. Salaffi F, Carotti M, Di Carlo M, Tardella M, Giovagnoni A. High-resolution computed tomography of the lung in patients with rheumatoid arthritis: prevalence of interstitial lung disease involvement and determinants of abnormalities. Medicine 2019;98:e17088.

30. Iannaccone CK, Lee YC, Cui J, Frits ML, Glass RJ, Plenge RM, et al. Using genetic and clinical data to understand response to disease-modifying anti-rheumatic drug therapy: data from the Brigham and Women's Hospital Rheumatoid Arthritis Sequential Study. Rheumatology 2011;50:40-6.

31. Solomon DH, Kremer J, Curtis JR, Hochberg MC, Reed G, Tsao P, et al. Explaining the cardiovascular risk associated with rheumatoid arthritis: traditional risk factors versus markers of rheumatoid arthritis severity. Ann Rheum Dis 2010;69:1920-5.

32. Aletaha D, Neogi T, Silman AJ, Funovits J, Felson DT, Bingham CO 3rd, et al. 2010 rheumatoid arthritis classification criteria: An American College of Rheumatology/European League Against Rheumatism collaborative initiative. Arthritis Rheum 2010;62:2569-81.

33. Arnett FC, Edworthy SM, Bloch DA, McShane DJ, Fries JF, Cooper NS, et al. The American Rheumatism Association 1987 revised 
criteria for the classification of rheumatoid arthritis. Arthritis Rheum 1988;31:315-24.

34. Anderson J, Caplan L, Yazdany J, Robbins ML, Neogi T, Michaud $\mathrm{K}$, et al. Rheumatoid arthritis disease activity measures: American College of Rheumatology recommendations for use in clinical practice. Arthritis Care Res 2012;64:640-7.

35. Pincus T, Keysor J, Sokka T, Krishnan E, Callahan LF. Patient questionnaires and formal education level as prospective predictors of mortality over 10 years in $97 \%$ of 1416 patients with rheumatoid arthritis from 15 United States private practices. J Rheumatol 2004;31:229-34.

36. Choi WI, Dauti S, Kim HJ, Park SH, Park JS, Lee CW. Risk factors for interstitial lung disease: a 9-year nationwide population-based study. BMC Pulm Med 2018;18:96.

37. Bankhead C, Silman A, Barrett B, Scott D, Symmons D. Incidence of rheumatoid arthritis is not related to indicators of socioeconomic deprivation. J Rheumatol 1996;23:2039-42.

38. Uhlig T, Hagen KB, Kvien TK. Current tobacco smoking, formal education, and the risk of rheumatoid arthritis. J Rheumatol 1999;26:47-54

39. Karlson EW, Daltroy LH, Lew RA, Wright EA, Partridge AJ, Fossel $\mathrm{AH}$, et al. The relationship of socioeconomic status, race, and modifiable risk factors to outcomes in patients with systemic lupus erythematosus. Arthritis Rheum 1997;40:47-56.

40. Di Giuseppe D, Discacciati A, Orsini N, Wolk A. Cigarette smoking and risk of rheumatoid arthritis: a dose-response meta-analysis. Arthritis Res Ther 2014;16:R61.
41. Fragoulis GE, Conway R, Nikiphorou E. MTX and interstitial lung disease: controversies and questions. A narrative review of the literature. Rheumatology 2019;58:1900-6.

42. Harlow L, Rosas IO, Gochuico BR, Mikuls TR, Dellaripa PF, Oddis $\mathrm{CV}$, et al. Identification of citrullinated HSP90 isoforms as novel autoantigens in rheumatoid arthritis-associated interstitial lung disease. Arthritis Rheum 2013;65:869-79.

43. Alunno A, Bistoni O, Pratesi F, La Paglia GMC, Puxeddu I, Migliorini P, et al. Anti-citrullinated alpha enolase antibodies, interstitial lung disease and bone erosion in rheumatoid arthritis. Rheumatology 2018;57:850-5.

44. Chen J, Song S, Liu Y, Liu D, Lin Y, Ge S, et al. Autoreactive T cells to citrullinated HSP90 are associated with interstitial lung disease in rheumatoid arthritis. Int J Rheum Dis 2018;21:1398-405.

45. Liu Y, Liu C, Li L, Zhang F, Li Y, Zhang S. High levels of antibodies to citrullinated $\alpha$-enolase peptide-1 (CEP-1) identify erosions and interstitial lung disease (ILD) in a Chinese rheumatoid arthritis cohort. Clin Immunol 2019;200:10-5.

46. White ES, Xia M, Murray S, Dyal R, Flaherty CM, Flaherty KR, et al. Plasma surfactant protein-D, matrix metalloproteinase-7, and osteopontin index distinguishes idiopathic pulmonary fibrosis from other idiopathic interstitial pneumonias. Am J Respir Crit Care Med 2016;194:1242-51.

47. Juge PA, Lee JS, Ebstein E, Furukawa H, Dobrinskikh E, Gazal $S$, et al. MUC5B promoter variant and rheumatoid arthritis with interstitial lung disease. N Engl J Med 2018;379:2209-19. 